\title{
Low-temperature Preparation of Mechanically Robust and Contamination-resistant Antireflective Coatings for Flexible Polymeric Glasses via Embedding of Silica Nanoparticles and HMDS Modification
}

Xiaodong Wang $\dagger^{\| *}$, Huiyue Zhao ${ }^{\dagger} \|$, Yixuan $\mathrm{Su}^{\dagger}$, Chen Zhang ${ }^{\dagger}$, Chen Feng ${ }^{\dagger}$, Qun Liu ${ }^{\star}$ and Jun Shen $\dagger^{* *}$

† Shanghai Key Laboratory of Special Artificial Microstructure Materials and Technology \& School of Physics Science and Engineering, Tongji University, Shanghai 200092, P. R. China

$\$$ College of Science, University of Shanghai for Science and Technology, Shanghai 200093, P. R. China

I| These authors contributed equally to this work.

\section{Corresponding Authors}

*E-mail: xiaodong_wang@tongji.edu.cn (X. Wang).

*E-mail: shenjun67@tongji.edu.cn (J. Shen).

\section{Contents}

TEM images of SNPs prepared with different amount of aqueous ammonia.

Optical property and hydrophobicity of PMMA coated with SNPs with different particle diameters.

Schematic diagram of the home-made chamber for chloroform vapor treatment.

Schematic diagram of the thermostat for water vapor treatment and HMDS vapor treatment.

Difference in embedding depth of SNPs by chloroform vapor treatment for different exposure time. 
Microstructure of SNP coatings and partial embedding of SNPs on PC substrates.

Surface quality of SNP coated PMMA before and after chloroform vapor treatment.

Optical transmittance of PMMA/PC coated with different coatings.

Optical performance of the SNP coatings coated on PC substrates.

Schematic diagram for simulation of "dirty" environment.

Mechanical and contamination-resistance properties of the SNP coatings coated on PC substrates. 

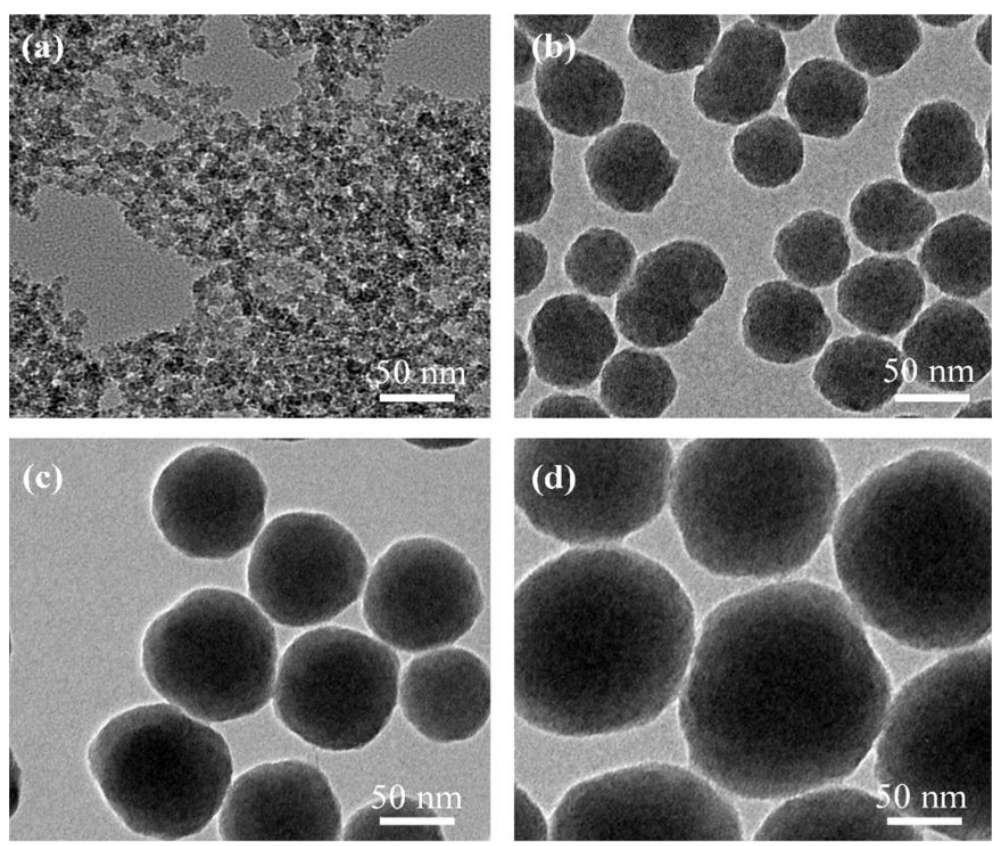

Figure S1. TEM images of SNPs prepared with different amount of aqueous ammonia: $5 \mathrm{ml}$ (a), $10 \mathrm{ml}(\mathrm{b}), 15 \mathrm{ml}(\mathrm{c})$ and $20 \mathrm{ml}(\mathrm{d})$.

Table S1 Optical property and hydrophobicity of PMMA coated with SNPs with different particle diameters.

\begin{tabular}{ccccc}
\hline Ammonia Volume/ ml & Diameter/ $\mathrm{nm}$ & $\mathrm{T}_{\max } / \%$ & $\begin{array}{c}\mathrm{T}_{\text {average }} / \% \\
(400-800 \mathrm{~nm})\end{array}$ & Water Contact Angle/ $^{\circ}$ \\
\hline 5 & 15 & 99.24 & 98.54 & $71.4 \pm 1.9$ \\
\hline 10 & 50 & 98.82 & 98.06 & $20.4 \pm 1.8$ \\
\hline 15 & 65 & 97.81 & 97.14 & $18.6 \pm 1.8$ \\
\hline 20 & 130 & 96.80 & 94.20 & $31.8 \pm 3.4$ \\
\hline
\end{tabular}




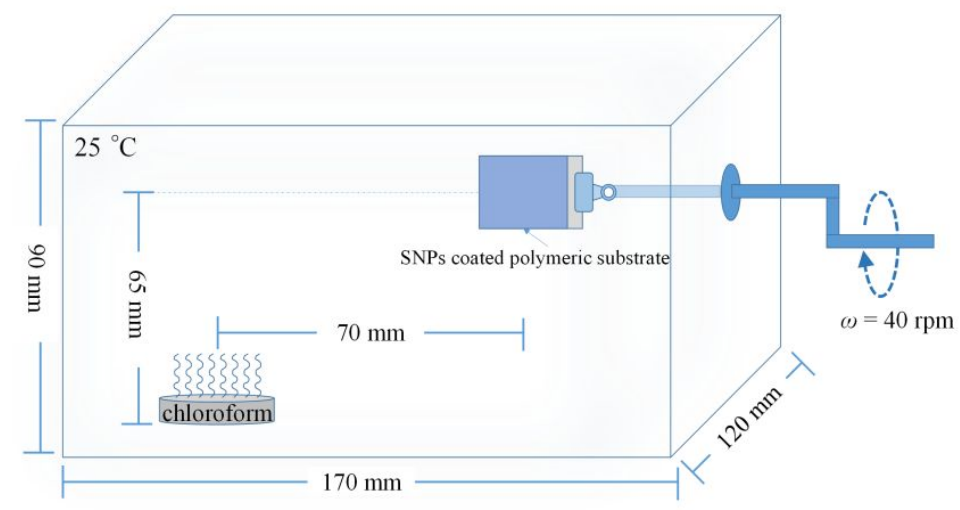

Figure S2. Schematic diagram of the home-made chamber for chloroform vapor treatment.

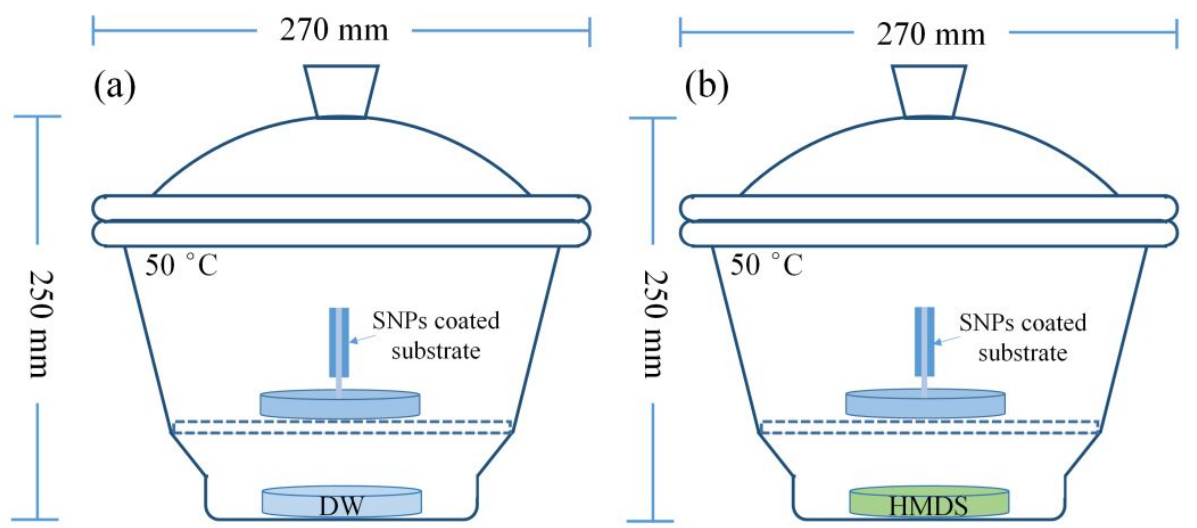

Figure S3. Schematic diagram of the thermostat for water vapor treatment (a) and HMDS vapor treatment (b). DW: deionized water, HMDS: hexamethyldisilazane. 
Difference in embedding depth of SNPs by chloroform vapor treatment for different exposure time.
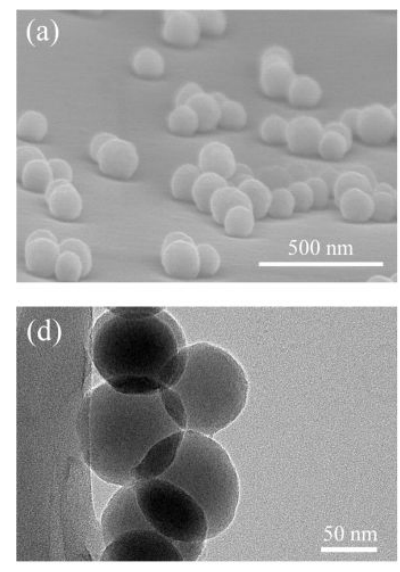
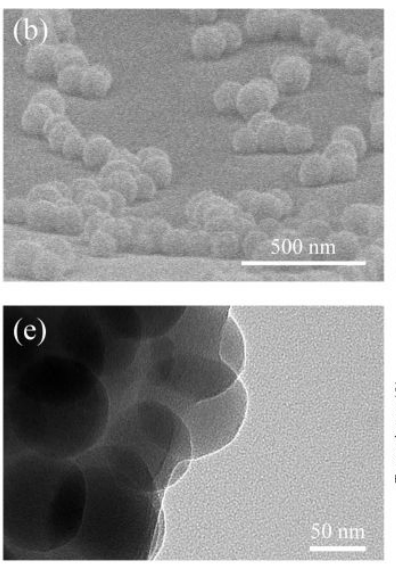
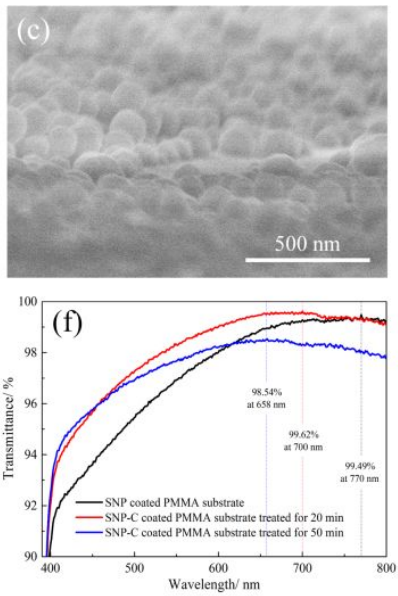

Figure S4. SEM images of SNP-C coated PC by chloroform vapor treatment for $3 \mathrm{~min}$ (a), $8 \mathrm{~min}$ (b), and 15 min (c); TEM images of SNP-C coated PC by chloroform vapor treatment for 3 min (d) and 15 min (e); Transmittance of SNPs coated PMMA by chloroform vapor treatment for different time (f).

The low conductivity of polymeric substrates and the thermal deformation induced by the highenergy electron beams limit the resolution of SEM imaging. Thus, it is difficult to directly perform high-resolution cross-sectional SEM images to accurately determine the embedding depth of the SNP coated substrates treated by $\mathrm{CHCl}_{3}$ vapor. Instead, we performed the FE-SEM imaging at an inclined angle to observe the difference in embedding depth. Due to the resolution limitation, larger SNPs prepared with high concentration of ammonia were separately dispersed on the substrate and treated by $\mathrm{CHCl}_{3}$ vapor for the easier SEM characterization of embedding. It can be distinguished from Figure S4 (a)-(c) that the embedding depth increases with the exposure time to chloroform vapor. TEM images were also characterized to observe the difference in embedding depth of the samples treated by chloroform for different time, as shown in Figure S4 (d)-(e). It is clearly observed that the embedding depth of SNPs coated on PC by chloroform vapor treatment for $15 \mathrm{~min}$ is much larger than that for $3 \mathrm{~min}$. Moreover, the different embedding depth of the samples was also indirectly confirmed from the change in transmittance spectra of the SNP-C coating coated substrates. As shown in Figure S4 (f), the transmittance spectra of the SNP-C coated PMMA shift towards shorter wavelength with the increase of the time of chloroform vapor 
treatment. This blue-shift indicates that the optical thickness of the SNP-C coating decreases with the time of chloroform vapor treatment, which is induced by the increasing embedding depth of the SNPs. All these results can be used to confirm that the embedding depth of the SNPs can be adjusted through the exposure time to $\mathrm{CHCl}_{3}$ vapor.

Microstructure of SNP coatings and partial embedding of SNPs on PC substrates.
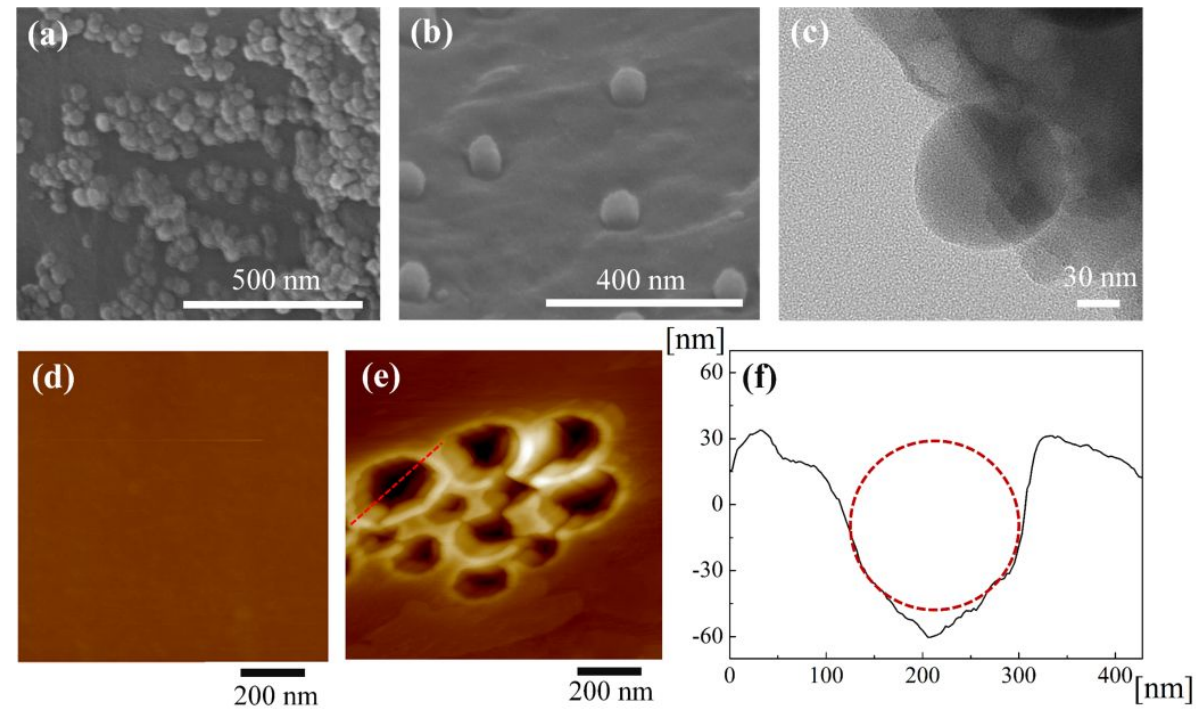

Figure S5. SEM images of SNPs fixed onto the PC substrate (a), SNPs embedded into the surface of PC substrate (b). TEM image of SNP embedded into the PC substrate (c). 2D AFM image of bare PC substrate (d), 2D AFM image (e) and height profile (f) of SNP coated PC substrate after HF etching. 
Surface quality of SNP coated PMMA before and after chloroform vapor treatment.
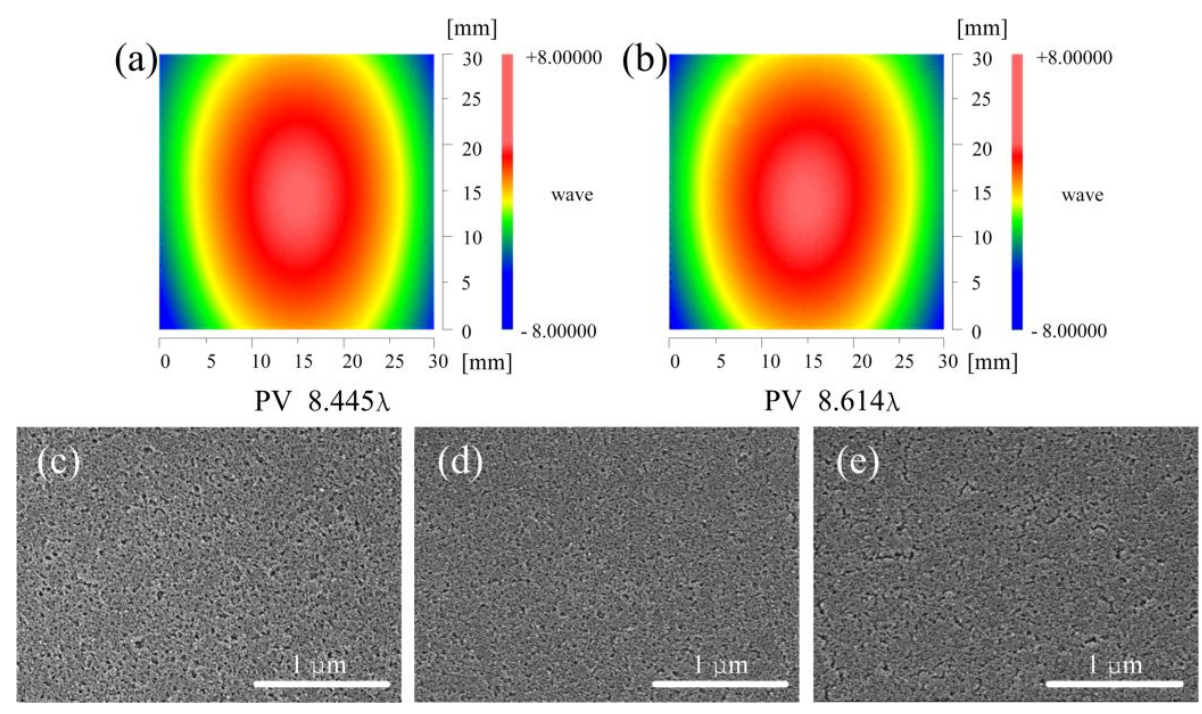

Figure S6. Surface profile of SNP coating coated PMMA before (a) and after chloroform vapor treatment for 20 min (b); SEM images of SNP coated PC substrate (c), after chloroform vapor treatment for $6 \min (\mathrm{d})$ and $9 \min (\mathrm{e})$.

Figure S6 (a)-(b) exhibit the surface profile of SNP coating coated PMMA before and after chloroform vapor treatment. The change in the surface figure (peak to valley (PV)) before and after chloroform vapor treatment is $0.169 \lambda$, which is much less than the PV of the coated PMMA substrate. The surface microstructure of the SNP coating before and after $\mathrm{CHCl}_{3}$ vapor treatment are also characterized with SEM and displayed in Figure S6 (c)-(e). As can be seen, the surface morphology of the SNP coating still presents a highly porous surface with nano pores and has no obvious difference after $\mathrm{CHCl}_{3}$ vapor treatment. This is due to the $\mathrm{CHCl}_{3}$ vapor treatment performed on the SNP coated polymeric substrate was a very mild and uniform treatment, while the treatment time was strictly controlled. In this way, the $\mathrm{CHCl}_{3}$ vapor treatment only will slightly made the SNPs partially embed into the substrates. Besides, the SNP coating deposited for antireflection purpose consists of multi-layers of SNPs stacked structure to meet the thickness requirement for antireflection in the visible wavelength range, rather than mono-particle layer. 
Only the bottom layer of SNPs were uniformly embedded into the substrate, which will not affect the surface morphology of the whole coating.

Table S2 Optical transmittance of PMMA/PC coated with different coatings.

\begin{tabular}{llll}
\hline Type of Substrate & & PMMA & PC \\
\hline & Bare Substrate & 92.30 & 89.20 \\
\cline { 2 - 4 } $\mathrm{T}_{\text {average }} / \%$ & SNP Coated & 98.54 & 96.76 \\
\cline { 2 - 4 }$(400-800 \mathrm{~nm})$ & SNP-C Coated & 98.81 & 97.38 \\
\cline { 2 - 4 } & SNP-CWH Coated & 98.62 & 97.63 \\
\hline \multirow{2}{*}{$\mathrm{T}_{\max } / \%$} & Bare Substrate & 92.70 & 90.44 \\
\cline { 2 - 4 } & SNP Coated & 99.39 & 98.06 \\
\cline { 2 - 4 } & SNP-C Coated & 99.75 & 99.27 \\
\cline { 2 - 4 } & SNP-CWH Coated & 99.32 & 98.84 \\
\hline
\end{tabular}

Optical performance of the SNP coatings coated on PC substrates.
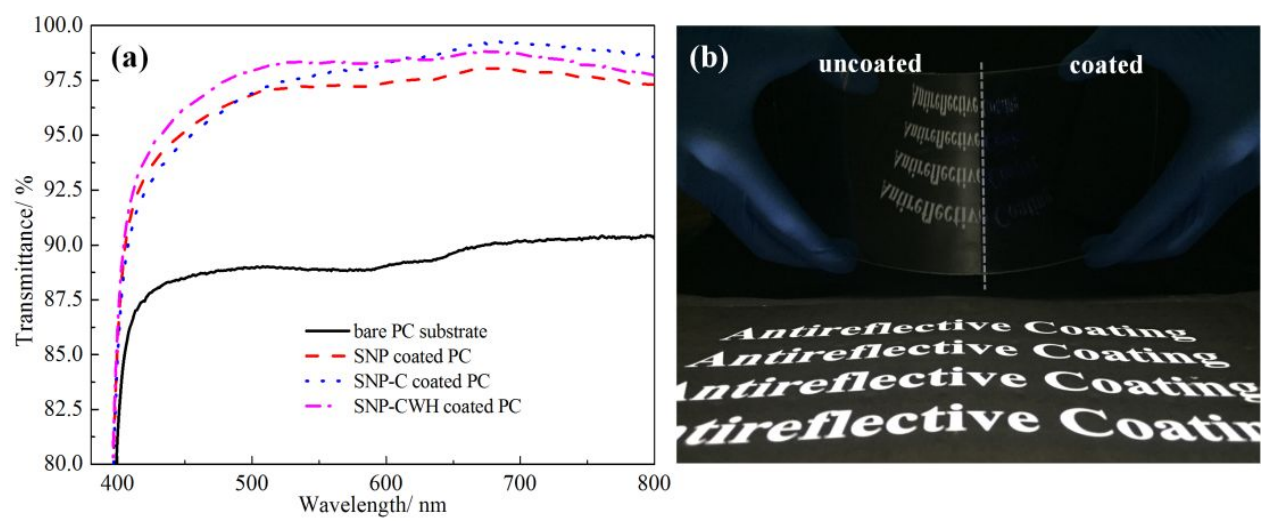

Figure S7. Transmittance spectra of SNP, SNP-C and SNP-CWH coated PC substrate (a) and the photograph of SNP-CWH coating on a bended PC substrate (b). 


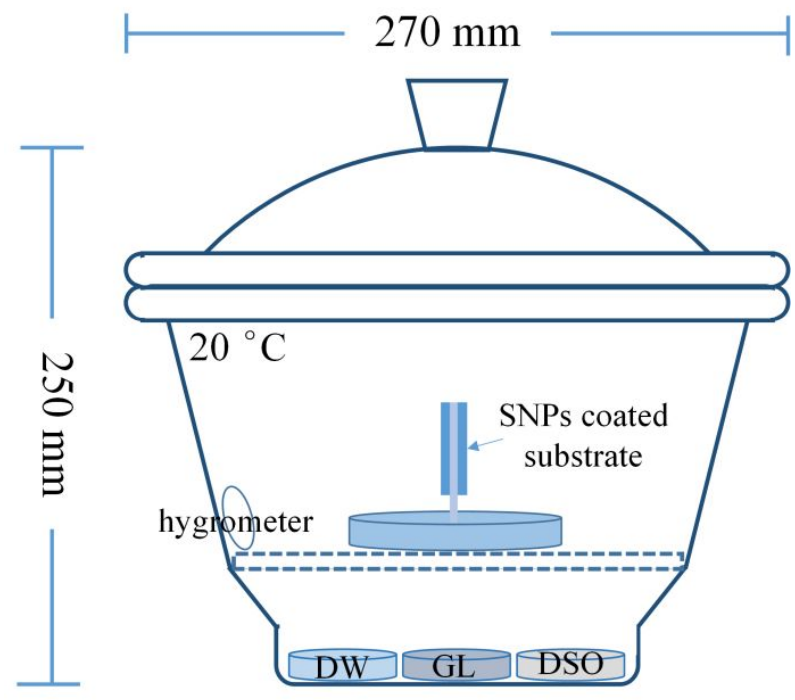

Figure S8. Schematic diagram of the chamber for simulation of "dirty" environment. DW: deionized water, GL: glycerol, DSO: dimethyl silicone oil.

$10 \mathrm{ml}$ of glycerol and $10 \mathrm{ml}$ of dimethyl silicone oil were put into the chamber as the organic contaminants. Meanwhile, $20 \mathrm{ml}$ of deionized water was put into the sealed chamber to create a high humid environment $(\mathrm{RH} \geqslant 90 \%)$, while a hygrometer was also put aside the sample to monitor the environment humidity. During the test, the condition was always re-set to the above parameters every time after the sample was taken out for the transmission characterization and was sent back immediately. 
Mechanical and contamination-resistance properties of the SNP coatings coated on PC substrates.
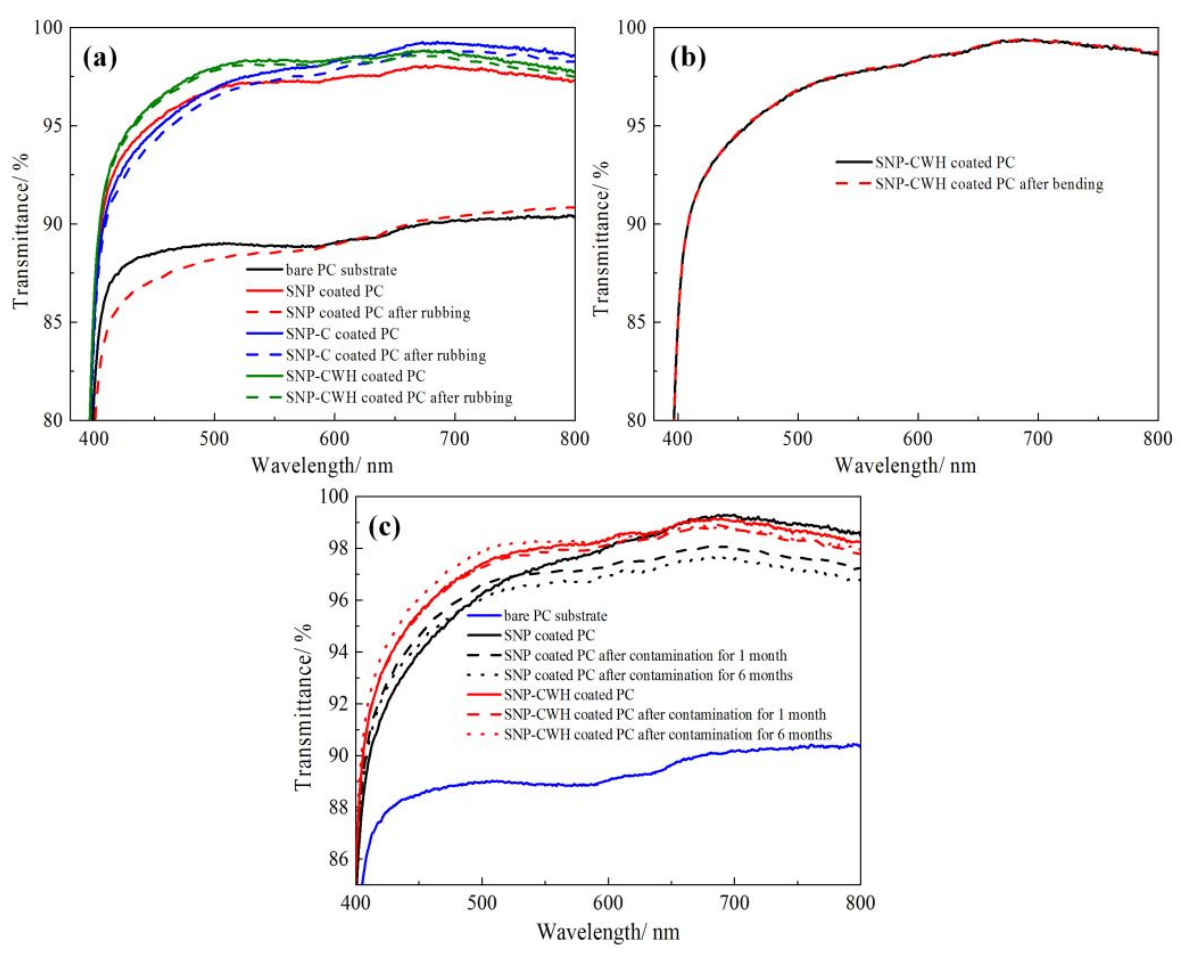

Figure S9. Transmittance spectra of the SNP, SNP-C and SNP-CWH coated PC substrate before and after rubbing for 100 times (a). Transmittance of the SNP-CWH coated PC substrate before and after bending for 50 times (b). Transmittance spectra of PC substrate coated with different coatings before and after contamination under a "dirty" environment for different periods (c). 